\title{
Performances en croisement et facteurs de variation des ovins Beni Guil au Maroc. I. Caractères de reproduction de la brebis et de viabilité et de croissance pré-sevrage des agneaux
}

\author{
M. El Fadili 1
}

\section{Mots-clés}

Ovin - Lignée pure - Croisement Reproduction - Viabilité -

Croissance - Maroc.

\begin{abstract}
Résumé
Les performances de reproduction des brebis, de viabilité et de croissance des agneaux ont été analysées pour les années 1994 à 1998 sur 484 brebis et 461 agneaux issus de l'accouplement de la race Béni Guil en race pure et en croisement avec les béliers de race D'man, Ile-de- France et Lacaune, à la station expérimentale El Koudia de I'Inra. Les résultats ont montré que la race du bélier a eu un effet très significatif sur la fertilité, le poids de la portée et les performances pondérales de la naissance au sevrage, mais elle n'a eu aucune influence significative sur la taille de la portée et la viabilité des agneaux à la naissance et au sevrage. La race Beni Guil a réalisé des performances moindres, excepté pour les caractères de fertilité et de viabilité des agneaux. Les croisements de la brebis Beni Guil avec les béliers Ile-de-France et Lacaune ont donné les meilleurs poids, gains de poids et productivité pondérale. L'âge de la brebis et l'année de naissance n'ont pas eu d'effet significatif sur les caractères de reproduction, à l'exception du poids de la portée à 90 jours. A tout âge, les agneaux nés simples et de sexe mâle ont réalisé une croissance supérieure à celle des femelles. L'année de naissance a eu un effet important sur les performances pondérales des agneaux. II a été conclu que le croisement avec les béliers Ile-de-France et Lacaune améliore la productivité pondérale par brebis au Maroc.
\end{abstract}

\section{INTRODUCTION}

Au Maroc, l'élevage ovin se chiffre à plus de 17,3 millions de têtes et joue un rôle important dans l'économie nationale. Ce cheptel est dominé, à plus de 95 p. 100, par des races locales rustiques de parcours. Ces dernières se caractérisent principalement par une faible prolificité des brebis (3) et une croissance modeste de leurs agneaux. Depuis le début des années 1990, le développement du

1. Unité de production animale et fourrages, Crra-Rabat/Inra, BP 415, Rabat, Maroc. Tél. : +212737675098; fax : +212737675098

E-mail: elfadili.moussa@yahoo.com croisement industriel pour la production d'agneaux précoces avec une carcasse conformée connaît de plus en plus d'intérêt. Les études sur l'utilisation des races de croisement terminal, Ile de France, Suffolk, Mérinos précoce et Lacaune, avec les races pures Timahdite, Sardi et Béni Guil (2, 3, 6, 7), ont montré une amélioration importante de la productivité de la brebis, et de la croissance et de la qualité de carcasse des agneaux. Dans cette étude ont été quantifiées les performances de reproduction de la brebis, de viabilité et de croissance des agneaux en race pure Beni Guil et aussi en croisement avec les races paternelles D'man, Ile-de-France et Lacaune, rameau viande, au domaine expérimental El Koudia de l'Institut national de la recherche agronomique (Inra). 


\section{MATERIEL ET METHODES}

\section{Animaux et modalités d'accouplement}

L'expérimentation s'est déroulée durant les années 1994-98 à la station El Koudia, située à 30 kilomètres au sud est de Rabat, à $150 \mathrm{~m}$ d'altitude, sur la côte atlantique. Le climat est semi-aride avec des précipitations annuelles moyennes variant de 350 à $500 \mathrm{~mm}$ et réparties entre novembre et mai.

La race Beni Guil (BG) fait partie des principales races locales de parcours concernées par le programme national d'amélioration génétique. Elle peuple les zones des plateaux de l'Oriental du Maroc, de Tendrara, Bouarfa, Figuig. Elle valorise particulièrement les plateaux et les steppes de cette région mais elle est capable de s'acclimater ailleurs au Maroc. Son effectif est estimé en 1996 à 1910881 têtes, soit 11,42 p. 100 du cheptel national ovin (11). La race $B G$ est de taille moyenne et sa queue est fine. La tête est nue, brune foncée chez le mâle et légèrement plus claire chez la femelle. Cette coloration s'étend jusqu'à l'arrière des cornes et à la mâchoire inférieure. Les membres sont également nus, de couleur brun feu ou marron. Chez le mâle, le profil et le chanfrein sont busqués et les cornes sont régulières et bien ouvertes en spirale. La femelle est motte et le chanfrein est plutôt droit ou légèrement busqué et la toison est blanche. Le animaux (brebis et béliers) utilisés ont été achetés en 1994 chez les éleveurs de l'Anoc et au niveau des souks situés dans le berceau de la race BG.

La race D'man (D) est connue pour ses caractéristiques reproductives exceptionnelles de prolificité, de précocité sexuelle et d'absence d'anœstrus (1). Cependant, le mode de conduite spécifique au système d'élevage oasien de cette race, sa mauvaise adaptation aux conditions de pâturage et la faiblesse de ses effectifs (inférieurs à 4 p. 100) dans la population totale de moutons au Maroc suggèrent son utilisation en croisement (race de bélier) en vue d'améliorer la productivité numérique des autres races locales beaucoup moins prolifiques. L'utilisation de béliers D'man dans cette étude a permis surtout d'apprécier les performances de croissance et de viabilité des agneaux issus du croisement D'man x Béni Guil et celles de Béni Guil purs. Les béliers D'man provenaient de la station expérimentale de l'Inra à Errachidia.

La race Ile-de-France (IF) est connue pour sa bonne croissance et sa conformation. Elle est très utilisée au Maroc en croisement industriel depuis les années 1950. La race Lacaune issue du rameau viande (LC) a été introduite à la station expérimentale El Koudia pour être testée comme une race alternative dans le croisement terminal, compte tenu de son potentiel de croissance élevé et aussi de son origine laitière et rustique du sud de la France. Les béliers

\section{Tableau I}

Répartition du nombre de brebis par type d'accouplement et par âge

\begin{tabular}{lcccc} 
Age & BG & D x BG & LC $\times$ BG & IF x BG \\
\hline Age $\leq 3$ ans & 18 & 47 & 13 & 92 \\
$3<$ âge $\leq 4$ & 60 & 32 & 14 & 12 \\
$4<$ âge $\leq 5$ & 54 & 28 & 16 & 17 \\
$5<$ âge $\leq 6$ & 40 & 22 & 15 & 18 \\
Age $>6$ ans & 15 & 20 & 14 & 15
\end{tabular}

BG : Beni Guil ; D : D'man ; LC : Lacaune ; IF : Ile de France améliorés IF et LC utilisés ont été achetés chez les éleveurs sélectionneurs de l'Anoc.

La répartition du nombre de brebis par type d'accouplement et par âge ainsi que celle des agneaux par génotype, par sexe et par type de naissance est montrée dans les tableaux I et II.

\section{Conduite d'alimentation des animaux}

Les brebis dans les différents accouplements ont été élevées dans des conditions de conduite et d'alimentation similaires selon un rythme de reproduction d'un agnelage par an. Les antenaises ont été mises à la lutte en bergerie, à l'âge moyen de 1,5 an, en été, pour une période de 45 jours (j) en juillet et août. Les brebis ont été conduites toute l'année sur des pâturages du domaine constitués de jachères et de chaumes dans des troupeaux de grande taille. Les brebis ont reçu le soir en bergerie un complément alimentaire dont la composition variait selon les aliments disponibles (orge et triticale grain, paille, foin d'avoine, tourteau de tournesol et complément minéral vitaminé). La quantité du complément distribué a dépendu de l'état des pâturages et des besoins physiologiques des animaux (entretien, gestation, allaitement). Chaque année, un programme annuel de vaccination et de déparasitage a été appliqué à l'ensemble des animaux.

Les agneaux nés en décembre et janvier ont été identifiés et pesés dans les 12 premières heures qui ont suivi la naissance. En plus du lait maternel, ils ont eu accès à un complément alimentaire solide pour jeunes animaux. Les agneaux ont été pesés individuellement à la naissance, puis tous les vingt et un jours pendant cinq pesées successives. Les poids obtenus lors des différentes pesées ont été standardisés aux âges types de 10, 30 et 90 j par extrapolation linéaire entre deux pesées adjacentes. Les gains moyens quotidiens (Gmq) ont été calculés à partir des poids standardisés.

\section{Caractères étudiés}

Chez la brebis, les caractères mesurés ont été la fertilité (nombre de brebis agnelées sur le nombre de brebis à la lutte), la taille et le poids de la portée à la naissance (TPN = nombre d'agneaux nés vivants ou morts par brebis agnelée PPN = poids de la portée par brebis agnelée), la taille de la portée au sevrage à $90 \mathrm{j}$ (TPS = nombre d'agneaux sevrés par brebis agnelée) et le poids de la portée au sevrage à $90 \mathrm{j}$ (PPS = poids total sevré par brebis agnelée). Chez l'agneau, les caractères contrôlés ont été la viabilité à la naissance durant les 12 premières heures et à $90 \mathrm{j}$ d'âge, le poids à la naissance, à 30 et à $90 \mathrm{j}$, et les Gmq entre 10 et $30 \mathrm{j}$ (Gmq10-30) et entre 30 et $90 \mathrm{j}$ (Gmq30-90). Tous les agneaux ont été évalués en terme de viabilité $(0=$ mort $; 1=$ vivant $)$.

\section{Tableau II}

Répartition du nombre d'agneaux par génotype, sexe et mode de naissance

\begin{tabular}{lcccc} 
Génotype & \multicolumn{2}{c}{ Sexe } & \multicolumn{2}{c}{ Mode de naissance } \\
\cline { 2 - 3 } & Mâle & Femelle & Simple & Double \\
\hline BG & 92 & 120 & 06 & 6 \\
D x BG & 64 & 58 & 194 & 18 \\
LC x BG & 31 & 32 & 58 & 5 \\
IF x BG & 38 & 26 & 58 & 6 \\
\hline
\end{tabular}

BG : Beni Guil ; D : D'man ; LC : Lacaune ; IF : Ile de France 


\section{Analyse statistique}

Les modèles d'analyse statistique ont inclus les principaux facteurs de variation contrôlés : le type d'accouplement de la brebis ou le génotype de l'agneau, l'âge de la brebis, l'année de naissance, le sexe et le type de naissance de l'agneau. Les modèles linéaires fixes définis ci-dessous ont été ajustés aux données en utilisant la procédure du modèle linéaire généralisé (13). Les modèles utilisés ainsi que les facteurs de variation retenus ont été :

- pour la fertilité, TPN, PPN, TPS et PPS :

$\mathrm{Y}_{\mathrm{ijkn}}=\mu+\mathrm{AC}_{\mathrm{i}}+\mathrm{AN}_{\mathrm{j}}+\mathrm{AG}_{\mathrm{k}}+\mathrm{e}_{\mathrm{ijkn}}$

- pour le poids à la naissance, 30 j, 90 j, Gmq10-30 et Gmq30-90 :

$\mathrm{Y}_{\mathrm{ijlmn}}=\mu+\mathrm{G}_{\mathrm{i}}+\mathrm{AN}_{\mathrm{j}}+\mathrm{S}_{1}+\mathrm{T}_{\mathrm{m}}+\mathrm{e}_{\mathrm{ijlmn}}$

où $\mathrm{Y}_{\mathrm{ijkn}}$ est la $\mathrm{n}^{\mathrm{e}}$ performance de la brebis d'âge $\mathrm{k}$, utilisée dans l'accouplement i durant l'année j ;

$\mathrm{Y}_{\mathrm{ijlmn}}$ est une performance du $\mathrm{n}^{\mathrm{e}}$ agneau de génotype $\mathrm{i}$, de sexe 1 , de type de naissance $m$ né durant l'année $\mathrm{j}$;

$\mu$ est la moyenne générale ;

$\mathrm{AC}_{\mathrm{i}}$ est l'effet fixe du type d'accouplement i (BG ; D x BG ; IF x $\mathrm{BG}, \mathrm{LC} \times \mathrm{BG})$;

$\mathrm{G}_{\mathrm{i}}$ est l'effet fixe du génotype de l'agneau i (BG ; D x BG ; IF x $\mathrm{BG}, \mathrm{LC} \times \mathrm{BG})$;

$\mathrm{AN}_{\mathrm{j}}$ est l'effet fixe de l'année de naissance j (1994 ... 1998);
$\mathrm{AG}_{\mathrm{k}}$ est l'effet fixe de l'âge de la brebis $\mathrm{k}$ (âge $\leq 3$ ans, $3<$ âge $\leq 4$, $4<$ âge $\leq 5,5<$ âge $\leq 6$, âge > 6);

$\mathrm{S}_{1}$ est l'effet fixe du sexe de l'agneau l (mâle, femelle);

$\mathrm{T}_{\mathrm{m}}$ est l'effet fixe du type de naissance de l'agneau m (simple, double) ;

$\mathrm{e}_{\mathrm{ijlmn}}$ est l'effet aléatoire résiduel.

\section{RESULTATS ET DISCUSSION}

\section{Performances de reproduction}

Les estimations des moindres carrés et leur erreur standard pour les effets type d'accouplement, année et âge de la brebis sur la fertilité, taille et poids de la portée à la naissance et à $90 \mathrm{j}$ sont présentées dans le tableau III.

\section{Fertilité et prolificité}

La fertilité et la prolificité à la naissance moyennes enregistrées ont été respectivement de 91 p. 100 et 1,05 agneau. Le type d'accouplement a eu un effet très significatif $(p<0,0001)$ sur la fertilité des brebis (tableau III). Accouplées en race pure ou en croisement avec les béliers $\mathrm{D}$, les brebis BG ont réalisé un taux de fertilité significativement supérieur à celles accouplées en croisement avec les béliers IF et LC. L'écart de fertilité entre les brebis accouplées aux béliers LC et IF a été de 2 p. 100. Boujenane et coll. (2) ont observé que la fertilité des brebis Timahdite et Sardi accouplées aux béliers IF était en moyenne inférieure de 5 p. 100

\section{Tableau III}

Moyennes des moindres carrés ( \pm erreur standard) des différentes performances par accouplement et selon l'âge de la brebis

\begin{tabular}{|c|c|c|c|c|c|c|}
\hline Source de variation & $\mathrm{Nb}$. & Fertilité (\%) & TPN (agneaux) & TPS (agneaux) & PPN (kg) & PPS (kg) \\
\hline Moyenne générale & & $91(27)$ & $1,05(0,21)$ & $0,98(0,31)$ & $3,65(0,76)$ & $19,86(6,44)$ \\
\hline Accouplement & & $* * *$ & NS & NS & $* * *$ & $* * *$ \\
\hline BG & 120 & $93 \pm 2^{a}$ & $1,07 \pm 0,02$ & $1,02 \pm 0,03$ & $3,60 \pm 0,08^{a}$ & $17,20 \pm 0,62 b$ \\
\hline$D \times B G$ & 216 & $94 \pm 2^{a}$ & $1,05 \pm 0,01$ & $0,98 \pm 0,02$ & $3,44 \pm 0,06^{b}$ & $18,94 \pm 0,50^{a}$ \\
\hline $\mathrm{LC} \times \mathrm{BG}$ & 73 & $80 \pm 3^{b}$ & $1,04 \pm 0,02$ & $0,97 \pm 0,04$ & $3,73 \pm 0,11^{b}$ & $22,32 \pm 0,87^{c}$ \\
\hline $\mathrm{IF} \times \mathrm{BG}$ & 75 & $78 \pm 3^{b}$ & $1,02 \pm 0,02$ & $0,93 \pm 0,04$ & $4,11 \pm 0,10^{b}$ & $21,79 \pm 0,86^{c}$ \\
\hline Age de la brebis & & NS & NS & NS & NS & $*$ \\
\hline Age $\leq 3$ ans & 92 & $88 \pm 4$ & $1,02 \pm 0,04$ & $0,92 \pm 0,05$ & $3,59 \pm 0,13$ & $19,93 \pm 1,15^{a b}$ \\
\hline $3<$ âge $\leq 4$ & 118 & $93 \pm 8$ & $1,02 \pm 0,04$ & $0,88 \pm 0,10$ & $3,67 \pm 0,13$ & $19,37 \pm 1,13 a b$ \\
\hline $4<$ âge $\leq 5$ & 115 & $82 \pm 7$ & $1,03 \pm 0,08$ & $0,97 \pm 0,09$ & $3,53 \pm 0,23$ & $16,99 \pm 2,01 \mathrm{a}$ \\
\hline $5<$ âge $\leq 6$ & 95 & $84 \pm 8$ & $1,16 \pm 0,07$ & $1,04 \pm 0,09$ & $4,00 \pm 0,20$ & $23,74 \pm 1,69 b$ \\
\hline Age $>6$ ans & 64 & $84 \pm 6$ & $1,10 \pm 0,06$ & $1,05 \pm 0,09$ & $4,01 \pm 0,17$ & $21,69 \pm 1,46 b$ \\
\hline Année & & $* * *$ & NS & NS & NS & $* *$ \\
\hline 1994 & 56 & $70 \pm 5^{a}$ & $1,03 \pm 0,05$ & $1,02 \pm 0,08$ & $3,48 \pm 0,16$ & $17,14 \pm 1,04{ }^{a}$ \\
\hline 1995 & 109 & $79 \pm 9 a b$ & $1,03 \pm 0,07$ & $1,05 \pm 0,10$ & $3,69 \pm 0,25$ & $19,65 \pm 2,06^{a}$ \\
\hline 1996 & 127 & $88 \pm 6 b$ & $1,12 \pm 0,06$ & $0,96 \pm 0,09$ & $3,86 \pm 0,21$ & $22,96 \pm 1,95 b$ \\
\hline 1997 & 110 & $97 \pm 7 b$ & $1,03 \pm 0,05$ & $0,98 \pm 0,08$ & $4,09 \pm 0,19$ & $20,31 \pm 1,08 \mathrm{ab}$ \\
\hline 1998 & 82 & $97 \pm 6^{b}$ & $1,03 \pm 0,05$ & $0,85 \pm 0,07$ & $3,49 \pm 0,17$ & $22,77 \pm 1,64 b$ \\
\hline
\end{tabular}

BG : Beni Guil ; D : D’man ; LC : Lacaune ; IF : Ile de France

TPN : taille de la portée à la naissance ; TPS : taille de la portée à $90 \mathrm{j} ;$ PPN : poids de la portée à la naissance ; PPS : poids de la portée à $90 \mathrm{j}$ NS : $\mathrm{p}>0,5 ; * * \mathrm{p}<0,01 ; * * * \mathrm{p}<0,001$

Les valeurs dans une même colonne avec des lettres différentes sont significativement différentes au seuil de $5 \%$. 
comparée à celle des brebis accouplées aux béliers Mérinos précoces. Le faible taux obtenu avec l'accouplement des brebis avec les béliers IF a également été observé dans le croisement avec les brebis Timahdite, élevées dans les mêmes conditions (5). En effet, les auteurs ont rapporté une fertilité inférieure de 7 à 8 p. 100 chez les brebis accouplées aux béliers IF en comparaison avec celles accouplées avec les béliers Mérinos précoces. La fertilité des brebis BG est comparable, en race pure, à celle des brebis Timahdite (89-91 p. 100), Sardi (91 p. 100) et D (91 p. 100), mais supérieure à celle des brebis Timahdite et Sardi croisées avec des béliers D (83-86 p. 100 et 82 p. 100, respectivement), et inférieure aux résultats du croisement des brebis Timahdite (91-93 p. 100) et Sardi (88 p. 100) avec les béliers LC et IF $(4,6)$.

La prolificité étant un caractère de la brebis, le type d'accouplement n'a pas eu d'effet significatif ( $\mathrm{p}>0,05$ ) sur la taille de la portée à la naissance et à $90 \mathrm{j}$ (tableau III). La TPN des brebis BG, quelle qu'ait été la race du bélier, s'est située entre 1,02 et 1,07 à la naissance, et entre 0,93 et 1,02 au sevrage à $90 \mathrm{j}$. La prolificité reste cependant comparable à celle des brebis Timahdite $(<1,20$ et $<1,14)$ et des brebis Sardi $(<1,09$ et $<1,0)$, respectivement à la naissance et à $90 \mathrm{j}(4,6)$.

Le PPN a été significativement plus faible $(\mathrm{p}<0,05)$ chez les brebis BG en race pure, mais les brebis accouplées aux béliers du croisement terminal ont enregistré le PPN le plus élevé, suivies par celles accouplées aux béliers D. De même, le PPS des brebis BG a été significativement supérieur $(\mathrm{p}<0,05)$ dans les croisements avec les béliers LC $(22,32 \mathrm{~kg})$ et IF $(21,79 \mathrm{~kg})$ en comparaison avec l'accouplement en race pure $(17,20 \mathrm{~kg})$ ou avec les béliers D $(18,94 \mathrm{~kg})$. Dans les mêmes conditions d'élevage, les portées sevrées des brebis Timahdite et Sardi ont été respectivement de 21,70 et $20,60 \mathrm{~kg}$ en race pure, de 21,89 et 20,20 kg en croisement avec les béliers D, et de 25,19 et $24,19 \mathrm{~kg}$ avec les béliers du croisement terminal (4).

La faible productivité pondérale (PPS) de la BG en comparaison avec la Timahdite et la Sardi, élevées dans les mêmes conditions de la station et avec les mêmes races de béliers de croisement, peut être expliquée en partie par le fait que les animaux (brebis et béliers) BG ont été achetés en majorité dans les souks, alors que les brebis Timahdite et Sardi ont été sélectionnées au niveau de la station El Koudia de l'Inra depuis plusieurs années.

\section{Effet de l'âge de la brebis}

L'âge de la brebis n'a pas eu d'influence significative $(\mathrm{p}>0,05)$ sur les caractères de fertilité et la taille de la portée à la naissance et au sevrage, mais son effet a été significatif $(p<0,05)$ sur le PPS. Les brebis jeunes ont enregistré des performances légèrement inférieures à celles des brebis âgées de plus de quatre années (tableau III). En général, tous les caractères de reproduction de la brebis ont eu tendance à augmenter avec l'avancement de l'âge de la brebis. Mavrogenis (12) a rapporté une diminution des performances des brebis à partir de la quatrième parité. El Fadili et coll. (6) ont observé que l'âge de la brebis a eu une influence significative sur les paramètres de reproduction lorsque les brebis étaient comparées dans différents accouplements en race pure et en croisement. Pour ces auteurs les performances maximales ont été réalisées par les brebis âgées de 4,5 ans.

\section{Effet de l'année}

L'année a eu une influence très significative sur la fertilité $(\mathrm{p}<$ $0,001)$ et sur le PPS $(p<0,01)$. Elle n'a pas eu d'effet significatif $(\mathrm{p}>0,05)$ sur la taille et le poids de la portée à la naissance et au sevrage. La fertilité et le PPS les plus faibles ont été observés en 1994 et 1995. Toutefois, l'effet de l'année semble être aléatoire et ne montre pas, en général, de tendance précise puisqu'il inclut des facteurs comme la disponibilité fourragère naturelle, les changements dans la gestion du troupeau et les conditions climatiques, particulièrement pour une conduite des animaux sur pâturage toute l'année, comme dans le cas de la présente étude. L'effet de l'année sur les performances de reproduction a été également rapporté par Boujenane et coll. (2).

\section{Viabilité et croissance des agneaux}

Les moyennes générales et les estimations de moindres carrées ainsi que leur erreur standard par facteur de variation sont rapportées dans le tableau IV.

\section{Effet du génotype}

Le génotype de l'agneau a eu un effet significatif $(\mathrm{p}<0,05)$ sur la viabilité des agneaux à la naissance mais a été sans effet à $90 \mathrm{j}$ d'âge (tableau IV). La viabilité des agneaux a été élevée et a varié de 94 à 100 p. 100 à la naissance et de 86 à 93 p. 100 au sevrage à $90 \mathrm{j}$. Le taux de viabilité observé chez les agneaux BG purs et croisés a été comparable à celui observé dans les croisements impliquant les races Timahdite et Sardi $(4,6)$. La viabilité légèrement inférieure des agneaux issus des croisements impliquant la race améliorée IF peut être expliquée en partie par l'apparition des mises bas dystociques, notamment chez les jeunes brebis donnant naissance à des agneaux plus lourds à la naissance. La mortinatalité élevée des agneaux à la suite du croisement de jeunes brebis avec les béliers de grand format a été aussi observée par Fahmy et coll. (9).

Le génotype de l'agneau a eu un effet très significatif $(\mathrm{p}<0,001)$ sur le poids et le Gmq des agneaux de la naissance au sevrage. A la naissance, les agneaux nés de pères IF ont été plus lourds, alors que les poids des agneaux issus de pères $\mathrm{D}$ et $\mathrm{BG}$ ont été comparables $(\mathrm{p}>0,05)$. Ceux nés de pères améliorés LC ont réalisé des poids intermédiaires (tableau IV). L'écart de poids entre les agneaux BG et croisés améliorés LC et IF s'est situé en moyenne à 0,26 et $0,62 \mathrm{~kg}$ à la naissance. La supériorité des croisés de pères IF et LC a été aussi observée pour les poids à 30 et 90 j et les Gmq10-30 et Gmq30-90. En effet, pour le poids au sevrage, les écarts entre les agneaux purs BG et les croisés LC et IF ont été respectivement de 4,07 et $3,93 \mathrm{~kg}$. Les agneaux LC ont réalisé des poids et Gmq qui n'étaient pas significativement différents $(\mathrm{p}>0,05)$, excepté à la naissance où la différence a été significative $(\mathrm{p}<0,05)$, comparés à ceux réalisés par les agneaux IF. Des observations similaires ont été faites dans les croisements impliquant les races Timahdite (6) et Sardi (2). La supériorité de la croissance des agneaux issus du croisement terminal semble être due aux différences dans les poids et gabarits adultes entre les races améliorées LC et IF et les races D et BG. Boujenane et coll. (2) ont rapporté également que les agneaux croisés de pères de races améliorées ont présenté une croissance rapide en comparaison à celle des agneaux des races pures Timahdite, Sardi et Béni Guil.

\section{Effet du sexe}

Le sexe a eu un effet significatif $(\mathrm{p}<0,05)$ sur la viabilité des agneaux à la naissance mais non significatif à $90 \mathrm{j}$ d'âge. Cependant, ce facteur de variation a eu une influence très significative $(\mathrm{p}<0,001)$ sur le poids et le gain de poids des agneaux à tout âge (tableau IV). Les agneaux mâles ont été toujours significativement plus lourds que les femelles de 0,26, 0,60 et 2,54 kg, respectivement à la naissance, à 30 et à $90 \mathrm{j}$. L'écart pour le Gmq a été en moyenne de $24 \mathrm{~g} / \mathrm{j}$ en faveur des mâles pour toute la période allant de la naissance au sevrage. La supériorité de la croissance des 


\section{Tableau IV}

Moyennes des moindres carrés ( \pm erreur standard) de la viabilité et de la croissance de la naissance à $90 \mathrm{j}$ des agneaux par génotype, sexe et type de naissance

\begin{tabular}{|c|c|c|c|c|c|c|c|c|}
\hline & Nb. & $\begin{array}{c}\text { Viab. naiss. }{ }^{1} \\
(\%)\end{array}$ & $\begin{array}{c}\text { Viab. à } 90 \text { j } \\
(\%)\end{array}$ & $\begin{array}{l}\text { Poids à naiss. } \\
\quad \text { (kg) }\end{array}$ & $\begin{array}{l}\text { Poids à } 30 \mathrm{j} \\
\quad(\mathrm{kg})\end{array}$ & $\begin{array}{l}\text { Poids à } 90 \mathrm{j} \\
\text { (kg) }\end{array}$ & $\begin{array}{c}\text { Gmq10-30 } 2 \\
(\mathrm{~g} / \mathrm{j})\end{array}$ & $\underset{(g / j)}{G m q 30-90}$ \\
\hline Moyenne générale & & $98(12)$ & $93(24)$ & $3,51(0,53)$ & $9,23(1,42)$ & $20,34(3,03)$ & $184(49)$ & $185(38)$ \\
\hline Génotype & & * & NS & $* * *$ & $* * *$ & $* * *$ & $* * *$ & $* * *$ \\
\hline BG & 212 & $99 \pm 1^{a}$ & $93 \pm 3$ & $3,12 \pm 0,06^{a}$ & $7,49 \pm 0,16^{a}$ & $17,24 \pm 0,35^{a}$ & $141 \pm 6^{a}$ & $162 \pm 4^{a}$ \\
\hline$D \times B G$ & 122 & $98 \pm 1^{\mathrm{a}}$ & $89 \pm 3$ & $3,07 \pm 0,05^{a}$ & $7,74 \pm 0,15^{a}$ & $17,53 \pm 0,32^{a}$ & $157 \pm 5^{a}$ & $165 \pm 4^{b}$ \\
\hline $\mathrm{LC} \times \mathrm{BG}$ & 63 & $100 \pm 2^{a}$ & $89 \pm 4$ & $3,38 \pm 0,08^{c}$ & $8,75 \pm 0,22^{b}$ & $21,31 \pm 0,47 b$ & $178 \pm 9 b$ & $209 \pm 6^{c}$ \\
\hline IF x BG & 64 & $94 \pm 2^{b}$ & $86 \pm 4$ & $3,80 \pm 0,08^{b}$ & $9,16 \pm 0,22 b$ & $21,17 \pm 0,48^{b}$ & $173 \pm 8^{b}$ & $200 \pm 6^{c}$ \\
\hline Sexe & & $*$ & NS & $* * *$ & $* *$ & $* * *$ & $* * *$ & $* * *$ \\
\hline Mâle & 225 & $99 \pm 2 b$ & $89 \pm 1$ & $3,47 \pm 0,03{ }^{a}$ & $8,59 \pm 0,15^{a}$ & $20,58 \pm 0,27^{a}$ & $169 \pm 5^{a}$ & $200 \pm 4^{a}$ \\
\hline Femelle & 236 & $97 \pm 2^{a}$ & $90 \pm 2$ & $3,21 \pm 0,06^{b}$ & $7,99 \pm 0,15^{b}$ & $18,04 \pm 0,26 b$ & $154 \pm 5 b$ & $168 \pm 4^{b}$ \\
\hline Type de naissance & & NS & $*$ & $* * *$ & $* * *$ & $* * *$ & $* * *$ & $* * *$ \\
\hline Simple & 416 & $98 \pm 1$ & $94 \pm 1$ & $3,63 \pm 0,03$ a & $9,50 \pm 0,08^{a}$ & $21,32 \pm 0,18$ & $188 \pm 3^{a}$ & $197 \pm 2^{a}$ \\
\hline Double & 45 & $98 \pm 2$ & $84 \pm 3$ & $3,05 \pm 0,08^{b}$ & $7,07 \pm 0,24 b$ & $17,30 \pm 0,53$ & $136 \pm 8^{b}$ & $171 \pm 7^{b}$ \\
\hline Année & & NS & NS & $* * *$ & $* * *$ & $* * *$ & $* * *$ & $* * *$ \\
\hline 1994 & 42 & $0,99 \pm 2$ & $0,93 \pm 0,04$ & $3,00 \pm 0,08^{a}$ & $7,20 \pm 0,26^{a}$ & $17,67 \pm 0,56^{a}$ & $142 \pm 6^{a}$ & $174 \pm 7^{a}$ \\
\hline 1995 & 100 & $0,98 \pm 1$ & $0,92 \pm 0,04$ & $3,37 \pm 0,08^{b}$ & $9,06 \pm 0,20^{b}$ & $21,96 \pm 0,42^{b}$ & $186 \pm 6^{b}$ & $215 \pm 5^{b}$ \\
\hline 1996 & 116 & $0,98 \pm 1$ & $0,89 \pm 0,03$ & $3,46 \pm 0,07^{b}$ & $8,84 \pm 0,18^{b}$ & $19,39 \pm 0,39^{c}$ & $183 \pm 6^{b}$ & $176 \pm 5^{a}$ \\
\hline 1997 & 12 & $0,99 \pm 1$ & $0,89 \pm 0,03$ & $3,51 \pm 0,06 b$ & $8,94 \pm 0,16^{b}$ & $19,34 \pm 0,34^{c}$ & $166 \pm 6^{c}$ & $173 \pm 4^{a}$ \\
\hline 1998 & 83 & $0,95 \pm 1$ & $0,82 \pm 0,03$ & $3,36 \pm 0,07 b$ & $7,40 \pm 0,20^{a}$ & $18,19 \pm 0,42 a$ & $144 \pm 6^{a}$ & $180 \pm 5^{a}$ \\
\hline
\end{tabular}

${ }^{1}$ Viabilité à la naissance $;^{2}$ gain de poids moyen quotidien de 10 à 30 jours

BG : Beni Guil ; D : D’man ; LC : Lacaune ; IF : Ile de France

NS : $p>0,05 ; * * p<0,01 ; * * * p<0,001$

Les valeurs dans une même colonne avec des lettres différentes sont significativement différentes au seuil de $5 \%$.

mâles sur les femelles a été également rapportée par El Fadili et coll. $(6,8)$ chez les agneaux Timahdite et croisés, et par Boujenane et coll. (2) chez les agneaux Sardi et croisés.

\section{Effet du type de naissance}

Le type de naissance a eu un effet important sur la viabilité des agneaux à $90 \mathrm{j}(\mathrm{p}<0,05)$ mais non significatif sur la viabilité à la naissance. Le type de naissance a eu également un effet important sur le poids à $30 \mathrm{j}$ et le Gmq10-30. Les agneaux nés simples ont été plus lourds de $0,60,2,43 \mathrm{~kg}$ et $52 \mathrm{~g} / \mathrm{j}$ our, respectivement pour le poids à la naissance, à $30 \mathrm{j}$ et le Gmq10-30. Au sevrage, les agneaux nés simples ont été plus lourds de 4,02 kg et leur Gmq30-90 a été supérieur de 32 g/jour. L'écart, moins important, du Gmq30-90 comparé au Gmq10-30 peut être expliqué par l'indépendance progressive des agneaux de leur mère mais aussi par leur ingestion d'aliments solides. L'effet important du type de naissance sur la croissance des agneaux a été aussi observé dans les recherches antérieures $(1,6,8)$.

\section{Effet de l'année}

L'année de naissance n'a pas eu d'effet significatif sur la viabilité des agneaux ( $p>0,05)$, mais son influence a été très significative $(\mathrm{p}<0,001)$ sur les poids et les $\mathrm{Gmq}$ individuels à tout âge (tableau IV). L'absence de l'effet année sur la viabilité des agneaux a été aussi observée par El Fadili et coll. (6). Les performances les plus faibles ont été réalisées par les agneaux durant les années 1994 et 1998, caractérisées par des précipitations plus faibles. La réduction de la productivité des pâturages a limité la production laitière des brebis, et ce, malgré le complément alimentaire apporté aux agneaux à l'auge. L'effet année sur les performances de croissance des agneaux a été aussi observé dans les travaux d'El Fadili et coll. (6,8), et Boujenane et coll. (2).

\section{CONCLUSION}

Les résultats de cette étude ont permis d'apporter des informations importantes pour contribuer à la caractérisation de la race Beni Guil, notamment en croisement. Les performances ont été obtenues sur des animaux au départ non sélectionnés et conduits dans des conditions de terrain très proches de celles de l'éleveur (pâturage toute l'année, troupeaux de grande taille). Les performances de la race Beni Guil en race pure ou avec les béliers des races D'man, Ile-de-France et Lacaune ont montré que, dans les conditions d'élevage de la station El Koudia, le croisement industriel avec les races améliorées Ile-de-France et Lacaune a donné les meilleurs résultats, excepté pour la fertilité des brebis et la viabilité des agneaux à $90 \mathrm{j}$. Les performances de croissance des agneaux croisés de pères améliorés ont été supérieures. Le croisement avec les béliers D'man, pour apprécier l'effet de la D'man, a donné des résultats comparables, voire supérieurs, à ceux obtenus en race pure Beni Guil pour le poids à $90 \mathrm{j}$ et le Gmq des agneaux. Au domaine expérimental El Koudia où de nombreux génotypes ont été comparés (3), les agneaux de la race Beni Guil ont présenté une croissance pré-sevrage inférieure à celle des agneaux des autres races locales rustiques. Ceci semble fortement lié aux poids et gabarit adultes 
moindres des Béni Guil qui présentent par ailleurs d'autres aptitudes intéressantes (rusticité, adaptation aux conditions arides et difficiles de la steppe de l'Oriental), mais qui semblent également posséder des qualités bouchères remarquables méritant des études ultérieures complémentaires.

\section{Remerciements}

L'auteur remercie le personnel de la station des ovins du domaine expérimentale El Koudia pour l'assistance technique. Cette recherche a été financée en partie par le ministère de l'Agriculture et de la Mise en valeur agricole du Maroc.

\section{BIBLIOGRAPHIE}

1. BERGER Y.M., BRADFORD G.E., ESSAADI A., JOHNSON D.W., BOURFIA M., LAHLOU-KASSI A., 1989. Performance of the D'man and Sardi sheep on accelerated lambing. III Lamb mortality, growth and production per ewe. Small Rumin. Res., 2: 307-321.

2. BOUJENANE I., BARADA D., MIHI S., JAMAI M., 1998. Reproductive performance of ewes and preweaning growth of lambs from three native Moroccan breeds mated to rams from Morocco and improved breeds. Small Rum. Res., 27: 203-208.

3. El FADILI M., 1996. Amélioration de la productivité des ovins par croisement. Rabat, Maroc, Institut national de la recherche agronomique, $62 \mathrm{p}$.

4. El FADILI M., LEROY P.L., 1997. Utilisation de la race $D^{\prime}$ man en croisement. In : Boulanouar B., Mattess-Guerrero A., Morkramer G., Ait Hroch A., éds, Actes de la journée d'étude sur la race D'man, Errachidia, Maroc, 9-12 déc. 1997, p. 63-80.

\section{Summary}

El Fadili M. Crossbreeding Performances and Variation Factors of Beni Guil Sheep in Morocco. I. Ewe Reproduction and Lamb Survival and Preweaning Growth Traits

An analysis of reproduction, survival and growth performances was performed on 484 Beni Guil ewes and their 461 progeny obtained by pure breeding or crossbreeding with D'man, Ile de France and Lacaune sires from data collected during 1994-98 at the experimental station El Koudia of INRA, Morocco. The results showed that the sire breed had a highly significant effect on the fertility, litter weight and growth from birth to weaning, but had no significant effect on the litter size at lambing, and on lamb survival at birth and weaning. The Beni Guil breed had the lowest performances, except for fertility and lamb survival. Crossbreeding between Beni Guil females and Ile de France and Lacaune terminal sires produced the highest weights, daily gains and productivity. The ewe age and birth year were not significant for reproduction traits except for the litter weight at 90 days. Regardless of the age, single-born males had higher growth rates than females. The birth year had a significant effect on lambs' weight performances. It was concluded that crossbreeding with lle de France and Lacaune sires improved weight productivity per ewe in Morocco.

Keywords: Sheep - Pure line - Crossbreeding - Reproduction Viability - Growth - Morocco.
5. El FADILI M., LEROY P.L., 2000. Comparaison de trois races de croisement terminal pour la production des agneaux croisés de boucherie au Maroc. Ann. Méd. vét., $145:$ 85-92.

6. El FADILI M., MICHAUX C., DETILLEUX J., LEROY P.L., 2000. Comparison of five crossbreeding types involving Timahdite, D'man and improved terminal sire breeds of sheep: ewe reproduction, lamb survival and growth performance. Anim. Sci., 71: 435-441.

7. EI FADILI M., MICHAUX C., LEROY P.L., 2000. Evaluation of fattening performances and carcass characteristics of purebred, first and second cross lambs between Moroccan Timahdite, D'man and improved meat rams. Anim. Sci., 72: 251-257.

8. EI FADILI M., MICHAUX C., BOULANOIR B., LEROY P.L., 2000. Environmental and genetic effects on growth in Timahdite and crossbred lambs in Moroco. Revue Elev. Méd. vét. Pays trop., 53 : 75-83.

9. FAHMY M.H., ROBERT S., GASTONGUAY F., 1997. Ewe and lamb behavior at parturition in prolific and non-prolific sheep. Can. J. Anim. Sci., 77: 9-15.

10. LAHLOU-KASSI A., BERGER Y.M., BRADFORD G.E., BOUKHLIQ R., TIBARY A., DERQAOUI L., BOUJENANE I., 1989. Performance of D'man and Sardi sheep on accelerated lambing. I. Fertility, litter size postpartum anoestrus and puberty. Small Rum. Res., 2: 225-239.

11. MADRPM, 1998. Présentation des résultats du recensement général de I'agriculture de 1996. Rabat, Maroc, Madrpm, direction de la Planification et des Affaires économiques.

12. MAVROGENIS A.P., 1996. Environmental and genetic factors influencing milk and growth traits of Awassi sheep in Cyprus. Heterosis and maternal effects. Small Rum. Res., 20: 59-65.

13. SAS, 2000. SAS/STAT user's guide, vers. 6, $4^{\text {th }}$ Ed. Cary, NC, USA, SAS Institute.

Accepté le 08.02.2010

\section{Resumen}

El Fadili M. Rendimientos en cruzamiento y factores de variación de ovinos de raza Beni Guil en Marruecos. I. caracteres de reproducción de la oveja y de viabilidad y crecimiento predestete de los corderos

En la estación experimental El Koudia del Inra, entre 1994 y 1998, se procedió al análisis del rendimiento reproductivo de las ovejas y de los parámetros de viabilidad y crecimiento de los corderos en 484 ovejas y 461 corderos resultantes del apareamiento de la raza Beni Guil en raza pura y en cruzamiento con carneros de las razas D'man, Ile de France y Lacaune. Los resultados mostraron que la raza del carnero tuvo un efecto muy significativo en la fertilidad, el peso de la camada y los rendimientos de peso del nacimiento al destete, pero no tuvo ninguna influencia significativa en el tamaño de la camada y la viabilidad de los corderos en el nacimiento y en el destete. La raza Beni Guil presentó un menor rendimiento, excepto en los caracteres de fertilidad y viabilidad de los corderos. Los cruzamientos de ovejas Beni Guil con carneros Ile de France y Lacaune proporcionaron los mejores pesos, ganancias de peso y productividad ponderal. La edad de la oveja y el año de nacimiento no tuvieron ningún efecto significativo en los caracteres de reproducción, con excepción del peso de la camada a los 90 días. En todas las edades, los corderos machos de parto simple presentaron un crecimiento superior al de las hembras. El año de nacimiento tuvo un efecto importante en los rendimientos de peso de los corderos. Se llegó a la conclusión de que el cruzamiento con carneros lle de France y Lacaune mejora la productividad ponderal por oveja en Marruecos.

Palabras clave: Ovino - Linea pura - Cruzamiento Reproducción - Viabilidad - Crecimiento - Marruecos. 